\title{
Biochimie des antinéoplasiques : le modèle du méthotrexate
}

L'inhibition classique de la dihydrofolate réductase par le méthotrexate n'explique pas seule son pouvoir cytolytique. Les dérivés polyglutamates du dihydrofolate et du méthotrexate, accumulés au cours du traitement par cette drogue, inhibent d'autres systèmes enzymatiques, ce qui a des conséquences sur l'utilisation thérapeutique du méthotrexate.

\section{Jacques Jolivet Francine Faucher Marie-France Pinard Patrice Dionne Richard Bertrand}

\section{ADRESSES}

J. Jolivet : institut du cancer de Montréal et hôpital Notre-Dame, 1560 rue Sherbrooke est, Montréal, H2L 4M1, Québec Canada.

F. Faucher, M.-F. Pinard, P. Dionne, R. Bertrand : institut du cancer de Montréal, 1560, rue Sherbrooke est, Montréal, H2L 4M1, Québec Canada.

$m / s n^{\circ} 4$ vol. 3 , avril 87 e méthotrexate (MTX) est un agent antinéoplasique employé depuis près de 40 ans dans le traitement d'une grande variété de cancers, y compris les leucémies de l'enfant, les tumeurs trophoblastiques, les lymphomes non hodgkiniens, les cancers du sein, de la tête et du cou, du poumon et les ostéosarcomes [1]. Le méthotrexate est également indiqué dans le traitement de maladies non cancéreuses, telles le psoriasis avancé, les rejets de greffes et l'arthrite rhumatoïde réfractaire. Il est également l'anticancéreux le plus étudié et dont on connaît le mieux les mécanismes biochimiques d'action et de résistance. Les premiers exemples d'amplification génétique ont été obtenus à partir de cellules résistantes au MTX. Plusieurs principes d'action biochimique des antimétabolites anticancéreux ont également été élucidés à partir d'études effectuées sur ce médicament. Ces connaissances biochimiques ont entraîné l'emploi clinique de hautes doses de MTX pour contrecarrer les mécanismes connus de résistance tumorale. Il est donc opportun de faire le point sur les

- $N A D P H=$ nicotinamide-adénine-dinucléotidephosphate réduit, coenzyme de la réductase. données récentes relatives aux mécanismes d'action et de résistance du MTX et d'examiner leurs implications sur la rationalité des protocoles cliniques employant de hautes doses de MTX.

\section{Mécanismes d'action}

Inhibition de la dihydrofolate réductase. Le MTX est un antifolate, c'est-à-dire un analogue 4- $\mathrm{NH}_{2}-10-\mathrm{CH}_{3}$ de l'acide ptéroylglutamique (acide folique ; PteGlu) (figure $1 B$ ), qui pénètre dans la cellule via une protéine transmembranaire spécifique utilisée normalement dans le transport des folates réduits [1]. Dès son entrée dans la cellule, le MTX se lie avec une grande affinité à l'enzyme dihydrofolate réductase (DHFR) qu'il inhibe en formant un complexe ternaire avec le $\mathrm{NADPH}^{*}$ [2]. Cette atteinte de la fonction de la DHFR est responsable des effets cytotoxiques du MTX car elle entraîne expérimentalement une inhibition de la synthèse de novo de l'acide thymidylique et des purines [3]. Traditionnellement, on explique cette inhibition de la façon suivante : durant la synthèse de novo de l'acide thymidylique, le méthylènetétrahydrofolate $\left(\mathrm{CH}_{2}-\mathrm{H}_{4}\right.$ PteGlu) est métabolisé en 


\section{RÉFÉRENCES}

1. Jolivet J, Cowan KH, Curt GA, Clendeninn NJ, Chabner BA. The pharnacology and clinical use of methotrexate. $N$ Engl $J$ Med 1983 ; 309 : 1094-104.

2. Matthews DA, Bolin JT, Burridge JM, Filman DJ, Volz KW, Krant J. Dihydrofolate reductase, the stereochemistry of inhibitor selectivity. J Biol Chem 1985 ; 260 : 392-9.

3. Werkheiser WC. The biochemical, cellular and pharnacological action and effects of the folic acid antagonists. Cancer Res 1963 ; 23 : 1277-85.

4. Allegra CJ, Fine RL, Drake JC, Chabner BA. The effect of methotrexate on intracellular folate pools in human MCF-7 breast cancer cells. Evidence for direct inhibition of purine synthesis. J Biol Chem 1986 ; 261 : 6478-85.

5. Kesavan V, Sur P, Doig MT, Scanlon KJ, Priest DG. Effects of methotrexate on folates in krebs ascites and L1210 murine leukemia cells. Cancer Lett $1986 ; 30$ : 55-9.

6. McGuire JJ, Bertino JR. Enzymatic synthesis and function of folylpolyglutamates. Mol Cell Biochem 1981; 38 : 19-48.

7. Taylor RT, Hanna ML. Folate-dependent enzymes in cultured Chinese hamster cells : folylpolyglutamate synthetase and its absence in mutants auxotrophic for glycine + adenosoine + thymidine. Arch Biochem Biophys 1977 ; 181 : 331-44.

8. Dolnick BJ, Cheng Y-C. Human thymidylate synthetase. Derivatives of pteroylmonoand-polyglutamates as substrates and inhibitors. $J$ Biol Chem 1978 ; 253 : 3563-7.

9. Allegra CJ, Drake JC, Jolivet J, Chabner BA. Inhibition of AICAR transformylase by methotrexate and dihydrofolic acid polyglutamates. Proc Natl Acad Sci USA 1985; 82 : 4881-5.

10. Allegra CJ, Drake JC, Jolivet J, Chabner BA. Inhibition of folate-dependent enzymes by methotrexate polyglutamates. In : Goldman ID, ed. Proceedings of the second workshop on folyl and antifolyl polyglulamates. New York : Praeger, 1985 : 348-59.

11. Chabner BA, Allegra CJ, Curt GA, at al. Polyglutamation of methotrexate. Is methotrexate a prodrug ? J Clin Invest 1985; 76 : 907-12.

12. McGuire JJ, Hsieh P, Coward JK, Bertino JR. Enzymatic synthesis of folylpolyglutamates : Characterization of the reaction and its products. J Biol Chem $1980 ; 255$ : $5776-88$

13. Jolivet J, Schilsky RL, Bailey BD, Drake JC, Chabner BA. The synthesis, retention and biological activity of methotrexate polyglutamates in cultured human breast cancer cells. $J$ Clin Invest $1982 ; 70: 351-60$.

dihydrofolate $\left(\mathrm{H}_{2}\right.$ PteGlu), un cofacteur inactif qui doit être réduit par la DHFR en tétrahydrofolate $\left(\mathrm{H}_{4} \mathrm{PteGlu}\right)$ pour redevenir un cofacteur à activité physiologique (figure 2). L'inhibition de la DHFR entraînerait donc théoriquement une accumulation intracellulaire de $\mathrm{H}_{2} \mathrm{PteGlu}$ et une déplétion des cofacteurs folates réduits. La synthèse de novo de l'acide thymidylique serait alors inhibée par des niveaux intracellulaires de $\mathrm{CH}_{2}-\mathrm{H}_{4} \mathrm{PteGlu}$ insuffisants, alors que deux étapes de la synthèse de novo des purines seraient inhibées par la diminution des niveaux de leur cofacteur, le $10-\mathrm{CHO}-\mathrm{H}_{4} \mathrm{PteGlu}$ (figure 2). La synthèse de l'ADN serait donc inhibée par manque de nucléotides. Ce schéma traditionnel vient d'être remis en question par des études récentes qui ont examiné directement les modifications des pools de folates intracellulaires secondaires à l'administration de MTX.

Rôle des dérivés polyglutamates - Folates intracellulaires. La mise au point de nouvelles techniques a permis récemment de mesurer avec précision les niveaux de folates intracellulaires $[4,5]$ : après exposition de cellules cancéreuses au MTX, les niveaux de $\mathrm{H}_{2}$ PteGlu, non détectables normalement, augmentent rapidement et représentent après environ une heure d'exposition près de $30 \%$ de tous les folates intracellulaires. Cette hausse se fait aux dépens des pools de $5-\mathrm{CH}_{3}$ $\mathrm{H}_{4}$ PteGlu, qui est le substrat de la méthionine synthase et habituellement la forme principale des folates intracellulaires. Les niveaux de $\mathrm{CH}_{2}-\mathrm{H}_{4}-$ PteGlu ainsi que de 10-CHO- $\mathrm{H}_{4}$ PteGlu diminuent à peine cependant et l'augmentation du $\mathrm{H}_{2}$ PteGlu cesse après une heure d'exposition au MTX. Le MTX n'entraîne donc pas de diminution importante des folates impliqués dans les synthèses de novo de l'acide thymidylique et des purines et, après une augmentation initiale, le substrat de la DHFR inhibée, le $\mathrm{H}_{2}$ PteGlu, cesse de s'accumuler. Puisque les synthèses de novo des purines et pyrimidines sont malgré tout stoppées, ces observations suggèrent qu'une inhibition directe de ces voies métaboliques est créée suite à l'inhibition de la DHFR par le MTX. Des études récentes sur le métabolisme intracellulaire des folates ont mis à jour les éléments nécessaires à une nouvelle compréhension d'action du MTX.

- Métabolisme intracellulaire des folates. Les folates intracellulaires existent sous forme de métabolites polyglutamates, c'est-à-dire de composés auxquels trois à six résidus $\gamma$-glutamyl supplémentaires ont été ajoutés au niveau de leur groupe glutamyl terminal par l'enzyme folylpolyglutamate synthétase (FPGS) [6] (figure 1A). Ce sont les formes physiologiquement actives des cofacteurs puisque des cellules mutantes sans FPGS sont incapables de survivre en culture cellulaire sans l'ajout de tous les produits des réactions où les folates agissent comme cofacteurs [7]. Les polyglutamates des folates ont une affinité accrue pour les enzymes dont ils sont des cofacteurs mais également pour d'autres enzymes impliquées dans le métabolisme des folates, dont ils peuvent être des inhibiteurs [6]. De plus, les répétitions "polyglutamates " portent plusieurs charges anioniques qui nuisent au transport de ces molécules hors de la cellule et assurent ainsi leur rétention cellulaire prolongée. Des études ont démontré que les polyglutamates du $\mathrm{H}_{2}$ PteGlu $\left(\mathrm{H}_{2}\right.$ PteGlu $\left.{ }_{n}\right)$ peuvent inhiber directement la thymidylate synthase [8] et les enzymes responsables de la synthèse de novo des purines $[9,10]$. Il est donc probable que l'augmentation des concentrations d' $\mathrm{H}_{2} \mathrm{PteGlu}_{\mathrm{n}}$ secondaire à l'inhibition de la DHFR par le MTX entraîne directement l'inhibition de ces voies enzymatiques. L'inhibition de la thymidylate synthase expliquerait également le plafonnement rapide des niveaux d' $\mathrm{H}_{2}$ PteGlu observé (figure 2).

- Métabolisme intracellulaire du MTX [11]. Alors que l'étude du métabolisme des folates permet $\mathrm{m} / \mathrm{s} n^{\circ} 4$ vol. 3, avril 87 
A.<smiles>CCC(=O)CCC(CCC(=O)O)NC(=O)c1ccc(N2C[C@@H]3CNc4nc(N)nc(O)c4N3P2)cc1</smiles>

B.

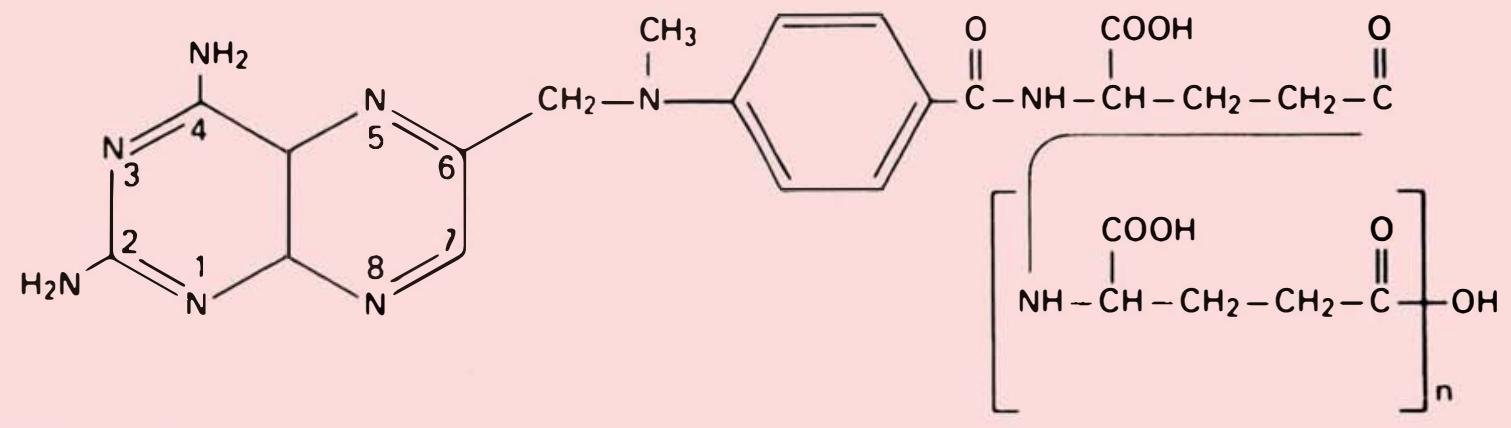

Figure 1. Structure des polyglutamates des tétrahydrofolates (A) et du méthotrexate (B). Les tétrahydrofolates (partie A de la figure) transportent les groupes monocarbonés (R) sur le $N^{5}$, le $N^{10}$ ou en formant un pont $N^{5}-10$.

Figure 2. Mécanisme d'action du méthotrexate. MTX = méthotrexate ; MTXPGs = polyglutamates du méthotrexate ; DHFR = dihydrofolate réductase ; TS = thymidylate synthase ; $\mathrm{H}_{2}$ PteGlu ${ }_{n}, \mathrm{H}_{4}$ PteGlu ${ }^{\prime}$ 5-10- $\mathrm{CH}_{2}-\mathrm{H}_{4}$ PteGlu et 10-CHO-H ${ }_{4}$ PteGlu $_{n}$ sont, respectivement, les polyglutamates du dihydrofolate, tétrahydrofolate, méthylènetétrahydrofolate et 10-formyltétrahydrofolate. Les lignes pointillées indiquent une inhibition enzymatique.

$\mathrm{m} / \mathrm{s} n^{\circ} 4$ vol. 3, avril 87

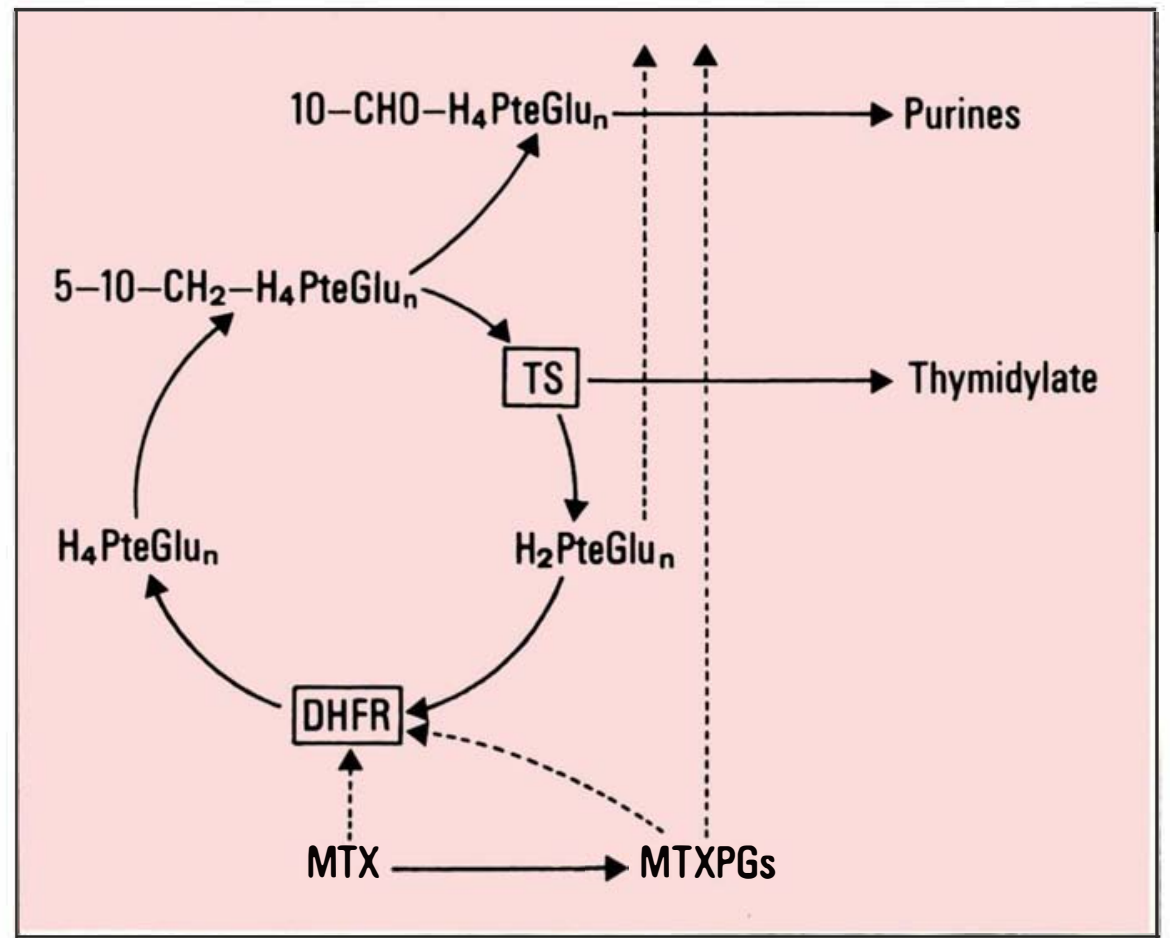


une meilleure compréhension des effets immédiats et précoces du MTX, des découvertes récentes sur le métabolisme intracellulaire du MTX ont permis d'élucider les mécanismes de certains effets cellulaires prolongés de l'antifolate. Comme les folates intracellulaires, le MTX est un substrat de l'enzyme folyl-polyglutamate synthétase [12]. Les dérivés polyglutamates du MTX (MTXPGs) (figure $1 B$ ) ont été identifiés dans plusieurs tissus animaux et humains exposés au MTX [1] : la synthèse des MTXPGs dépend de la concentration extracellulaire de MTX de même que de la durée de l'exposition à l'antifolate [13]. Ces métabolites conferent au MTX les caractéristiques supplémentaires suivantes : (a) une affinité accrue pour la DHFR[14]. Les MTXPGs remplacent le MTX sur la DHFR dès qu'ils apparaissent dans la cellule. Leur affinité pour l'enzyme cible est probablement supérieure à celle du MTX puisque les MTXPGs sont beaucoup plus difficilement dissociables de la DHFR que le MTX. (b) Une rétention intracellulaire. Comme les dérivés polyglutamates des folates, certains dérivés polyglutamates du MTX sont retenus beaucoup plus avidement dans les cellules que le MTX. Le MTX et les métabolites ayant un total de deux ou trois résidus glutamyls (MTXGlu $\mathrm{et}_{3}$ ) quittent les cellules de tumeurs mammaires humaines après leur transfert dans un milieu sans MTX, alors que les MTX-Glu sont retenus en partie et les MTX-Glu ${ }_{5}$ en totalité [13]. Ce phénomène est important puisque les MTXPGs peuvent persister dans la cellule à des concentrations suffisantes pour saturer la DHFR et inhiber la croissance cellulaire longtemps après la disparition du MTX extracellulaire [13-15]. (c) Une inhibition d'enzymes autres que la DHFR. L'addition de polyglutamate confere au MTX, comme aux folates physiologiques, une affinité accrue pour plusieurs enzymes dont les folates sont cofacteurs [6]. Traditionnellement, le MTX était considéré comme un inhibiteur spécifique de la DHFR avec un faible potentiel inhibiteur pour la thymidylate synthase, sans affinité significative pour aucune autre enzyme. Les MTXPGs cependant inhibent directement plusieurs autres enzymes $[9,10,16]$, dont la thymidylate synthase et certaines enzymes de la synthèse de novo des purines, à des concentrations intracellulaires de MTXPGs qui peuvent être atteintes au cours du traitement [13]. Il est donc probable que, comme les $\mathrm{H}_{2} \mathrm{PteGlu}_{\mathrm{n}}$, les MTXPGs puissent, in vivo, inhiber directement les synthèses de novo de l'acide thymidylique et des purines.

Le métabolisme du MTX prend toute son importance dans des expériences où l'exposition au MTX est de courte durée (24 heures ou moins), un schéma expérimental se rapprochant de la situation clinique [13-15]. C'est dans ces circonstances que la rétention prolongée des MTXPGs à longue chaîne glutamyl permet une inhibition de la DHFR qui se prolonge après disparition du MTX extracellulaire. Ceci entraîne probablement une élévation prolongée des niveaux d' $_{2} \mathrm{PteGlu}_{\mathrm{n}}$ qui, avec les MTXPGs, continuent à inhiber directement les synthèses de novo de l'acide thymidylique et des purines longtemps après l'administration du MTX (figure 2).

Mécanismes de cytotoxicité

Les mécanismes précis par lesquels l'inhibition de la DHFR et l'abolition qui en résulte de la synthèse de novo des purines et des pyrimidines sont cytotoxiques, restent inconnus. Des travaux encore préliminaires suggèrent que l'inhibition de la thymidylate synthase entraîne une élévation importante des niveaux intracellulaires de son substrat, l'acide désoxyuridylique triphosphate (dUTP) [17]. Celuici serait incorporé de façon anormale dans l'ADN [18], entraînant sa fragmentation et la mort cellulaire $[19,20]$. Ces résultats sont cependant contestés par certains chercheurs qui n'ont pas pu confirmer une telle incorporation de dUTP dans l'ADN à la suite
19. Ingraham HA, Dickeyl, Goulian M. DNA fragmentation and cytotoxicity from increased cellular deoxyuridylate. Biochemistry $1986 ; 25$ 
d'administration de MTX [21]. Néanmoins, cette hypothèse doit être explorée à fond car elle peut mener à une meilleure compréhension des mécanismes ultimes de la cytotoxicité au MTX.

\section{Mécanismes de résistance}

On reconnaît traditionnellement quatre mécanismes pour expliquer la résistance tumorale au MTX : (a) une altération du transport transmembranaire empêchant l'accumulation intracellulaire suffisante de MTX pour saturer la DHFR [22] ; (b) une augmentation des niveaux de DHFR empêchant le MTX de se lier à tous les sites enzymatiques [23]; (c) une altération de la DHFR caractérisée par une affinité réduite pour le MTX [24] ; (d) une diminution de la synthèse de novo de l'acide thymidylique rendant la cellule moins dépendante de l'activité de la DHFR et de ce fait moins sensible aux effets du MTX [25]. Un cinquième mécanisme s'est récemment ajouté à cette liste : un métabolisme insuffisant du MTX en MTXPGs. Trois approches expérimentales nous permettent de déduire que le métabolisme du MTX en MTXPGs est essentiel aux effets cytotoxiques du MTX : (a) il existe une corrélation directe entre la cytotoxicité du MTX et les quantités de MTXPGs formés dans plusieurs lignées tumorales expérimentales $[1,13,15]$; (b) une lignée humaine de cancer mammaire rendue résistante au MTX par exposition continue in vitro au médicament a comme principale anomalie une absence presque complète de synthèse de MTXPGs [26] ; (c) un analogue du MTX qui n'en diffère que par une substitution fluor dans le groupe terminal glutamyl (le $\gamma$ fluorométhotrexate) est beaucoup moins cytotoxique que le MTX [27].

Cet analogue ne peut pas être métabolisé en polyglutamates à cause du résidu fluor mais est un inhibiteur de la DHFR aussi efficace que le MTX, démontrant $m / s n^{\circ} 4$ ool. 3, avril 87 l'importance primordiale du métabolisme du MTX dans son mécanisme d'action.

\section{Hautes doses de MTX}

Bases biochimiques. Depuis plusieurs années, le MTX est employé à hautes doses, suivi de l'administration de 5-CHO$\mathrm{H}_{4}$ PteGlu (acide folinique) comme antidote pour les tissus normaux. Ces traitements ont été initialement mis au point pour surmonter une résistance tumorale au MTX secondaire à une anomalie du mécanisme de transport puisqu'on croyait que c'était le mécanisme principal de résistance à l'antifolate [1]. Expérimentalement, à hautes concentrations de MTX, une quantité suffisante de MTX peut pénétrer au travers de la membrane cellulaire anormale par diffusion et se lier à tous les sites actifs de la DHFR [22]. Malheureusement, dans certaines tumeurs [15], les autres mécanismes par lesquels une cellule cancéreuse résiste au MTX sont plus importants que les anomalies de transport et ne sont pas tous également réversibles par de hautes concentrations de MTX : (a) des niveaux élevés de DHFR ne sont pas nécessairement saturés par de hautes concentrations intracellulaires de MTX puisque le MTX lui-même peut induire, par des mécanismes encore mal connus, une surélévation aiguë du niveau de la DHFR dont le gène est déjà amplifié [23] ; (b) des DHFR avec affinité diminuée pour le MTX identifiées dans des cellules blastiques de leucémies myéloïdes aiguës humaines ont si peu d'affinité pour le médicament que l'activité enzymatique ne serait pas nécessairement inhibée par de hautes concentrations de MTX [24] ; (c) des lignées tumorales humaines de cancer du poumon à petites cellules indifférenciées, résistantes au MTX du fait d'une activité diminuée de la thymidylate synthase, sont peu sensibles à de hautes concentrations de MTX [15] ; (d) l'exposition de cellules tumorales à de hautes doses de MTX peut par- fois, mais pas toujours, outrepasser une résistance au MTX due à un métabolisme insuffisant en polyglutamates $[15,26]$.

Les hautes doses de MTX doivent être administrées en association avec l'acide folinique pour prévenir les effets toxiques accrus de cette forme de traitement. Traditionnellement, on reconnaît deux mécanismes par lesquels l'acide folinique peut agir comme antidote : (a) le folate peut inhiber le transport du MTX dans la cellule puisque les deux empruntent la même protéine de transport transmembranaire [1]. Ce mécanisme est probablement responsable de l'effet de l'acide folinique en présence du MTX et explique qu'expérimentalement, à doses trop élevées ou rapprochées du MTX, l'antidote peut abolir l'effet antitumoral de l'antifolate [28] ; (b) l'acide folinique peut reconstituer directement les pools intracellulaires de folates réduits qui auraient été déplétés sous l'effet du MTX. Cette dernière hypothèse doit être modifiée puisqu'on sait maintenant que le MTX affecte peu les pools de folates réduits impliqués dans la synthèse de novo des purines. Elle peut peut-être expliquer cependant l'observation expérimentale que l'acide folinique est un antidote compétitif du MTX, c'est-à-dire que plus la dose de MTX est élevée, moins l'antidote est efficace et, à doses très élevées de MTX, il est complètement incapable de réverser les effets de l'antifolate [29]. En effet, les pools de folates réduits augmentés à la suite de l'emploi de l'acide folinique serviront à réverser l'inhibition directe des enzymes des synthèses de novo des purines et des pyrimidines par les $\mathrm{H}_{2}$ PteGlu ${ }_{n}$ et les MTXPGs. A doses élevées de MTX, et par conséquent à des concentrations intracellulaires élevées de MTXPGs, les pools de folates quoiqu'accrus seront potentiellement insuffisants pour réverser ces inhibitions enzymatiques directes. De plus, certains folates réduits peuvent dissocier directement le MTX de la DHFR et rétablir 


\section{RÉFÉRENCES}

20. Li JC, Kaminskas E. Accumulation of DNA strand breaks and methotrexate cytotoxicity. Proc Nall Acad Sci USA 1984 ; 81 : 5694-8.

21. Fraser DC, Pearson CK. Is Uracil misincorporation into DNA of mammalian cells a consequence of methotrexate treatment ? Biochem Biophys Res Commun 1986 ; 135 : 886-93.

22. Hill BT, Dedhar S, Goldie JH. Evidence that at * high * extracellular methotrexate concentrations the transport barrier is unlikely to be an important mechanism of drug resistance. Biochem Pharmacol 1982 ; 31 : 263-6.

23. Cowan $\mathrm{KH}$, Goldsmith ME, Ricciardione M, Levine R, Rubalca E, Jolivet J. Regulation of dihydrofolate reductase in human breast cancer cells and in mutant hamster cells transfected with a human dihydrofolate reductase minigene. Mol Pharmacol 1986 ; 30 : 69-76.

24. Dedhar S, Hartley D, Fitz-Gibbons D, Phillips G, Goldie JH. Heterogeneity in the specific activity and methotrexate sensitivity of dihydrofolate reductase from blast cells of acute myelogenous leukemia patients. $J$ Clin Oncol $1985 ; 3$ : 1545-52.

25. Moran RG, Mulkins M, Heidelberg C. Role of thymidylate synthase activity in developement of methotrexate cytotoxicity. Proc Nall Acad Sa USA 1979 ; 76 : 5924-8.

26. Cowan KH, Jolivet J. A methotrexateresistant human breast cancer cell line with multiple defects, including diminished formation of methotrexate polyglutamates. $J$ Biol Chem 1984; 259 : 10793-800.

27. Galivan J, Inglese J, McGuire JJ, Nimec Z, Coward JK. $\gamma$-fluoromethotrexate : Synthesis and biological activity of a potent inhibitor of dihydrofolate reductase with greatly diminished ability to form poly- $\gamma$-glutamates Proc Nall Acad Sci USA 1985 ; 82 : 2598-602.

28. Sirotnak FM, Moccio DM, Dorick DM Optimization of high-dose methotrexate with leucovorin rescue therapy in the $L 1210$ leukemia and sarcoma 180 murine tumor models. Cancer Res 1978 ; 38 : 345-53.

29. Pinedo HM, Zaharko DS, Bull JM, Chabner BA. The reversal of methotrexate cytotoxicity to mouse bone marrow cells by leucovorin and nucleosides. Cancer Res 1976 ; 36 : 4418-24.

30. Matherly LH, Barlowe CK, Goldman ID. Antifolate polyglutamylation and competitive drug displacement at dihydrofolate reductase as important elements in leucovorin rescue in L1210 cells. Cancer Res 1986 ; 46 : 588-93.

31. Stoller RG, Kaplan HG, Cummings FJ Calabresi P. A clinical and pharmacological study of high-dose methotrexate with minimal leucovorin rescue. Cancer Res 1979; 39 . cette activité enzymatique [30]. Plus les sites enzymatiques de la DHFR seront cependant occupés par des MTXPGs, moins cette dissociation sera efficace, ce qui constitue une autre explication de l'inefficacité de l'antidote lors de l'administration de doses élevées de MTX.

Implications cliniques. L'emploi clinique de hautes doses de MTX n'a malheureusement réussi à passer outre la résistance tumorale au MTX que dans une minorité des cas et n'est encore utilisé régulièrement que dans le traitement des ostéosarcomes et des lymphomes non-Hodgkiniens [1]. Ceci n'est guère surprenant vu l'hétérogénéité des mécanismes de résistance au MTX et leur susceptibilité variable à une augmentation des concentrations de MTX. Plusieurs laboratoires tentent de développer des sondes biologiques qui permettront de doser les protéines impliquées dans la résistance au MTX et de n'employer ainsi les traitements par hautes doses de MTX que dans des situations appropriées. Il n'en demeure pas moins que, basées sur nos connaissances actuelles de la biochimie pharmacologique du MTX, certaines recommandations pratiques sur l'emploi du MTX à haute dose s'imposent : tout d'abord, il faut administrer le MTX, pour atteindre des concentrations plasmatiques de $10^{-4}$ à $10^{-5} \mathrm{M}$, pendant au moins vingtquatre heures. Ces concentrations de MTX sont suffisantes pour surmonter une résistance secondaire à un transport anormal, et la durée d'exposition permet d'optimiser la formation des dérivés polyglutamates du MTX. Une deuxième recommandation est d'administrer l'acide folinique à doses minimales et le plus longtemps possible après le MTX pour tenter d'éviter que l'antidote n'agisse sur les tissus cancéreux, diminuant alors l'effet thérapeutique du MTX. Des régimes d'administration du méthotrexate respectant ces principes existent déjà [31], mais ils méritent une étude clinique beaucoup plus approfondie

\section{Summary}

Recent concepts concerning the mechanism of action of the antifolate methotrexate (MTX) are presented. Traditionally, the drug has been thought to act solely by inhibiting dihydrofolate reductase, leading to depletion of reduced intracellular folates and consequent inhibition of de novo purine and pyrimidine synthesis. Recent studies have shown that the reduced folates involved in purine synthesis are not significantly affected by MTX and that the decreased nucleotide synthesis rate observed is probably the result of direct inhibition of these reactions by dihydrofolate polyglutamates which accumulate intracellularly after dihydrofolate reductase inhibition. The role of methotrexate polyglutamates in drug action is also discussed along with updated concepts of the mechanisms of MTX tumor resistance and their treatment with high-dose regimens.

\section{TIRÉS A PART}

J. Jolivet : institut du cancer de Montréal et hôpital Notre-Dame, 1560 rue Sherbrooke est, Montréal, H2L 4M1, Québec Canada. 